\title{
Evolution of Resistance to Fluoroquinolones of Uropathogenic Strains of Escherichia coli Isolated at the University Hospital of Marrakesh, Morocco
}

\author{
Saida Ed-dyb*, Khadija Ait Zirri, Fatima Babokh, Raihane Bahri, Asmaa Lamrani, Nabila Soraa \\ Microbiology Department, Mohammed VI University Hospital, Marrakesh, Morocco \\ Emailaddress: \\ saeddyb@gmail.com (S. Ed-dyb) \\ ${ }^{*}$ Correspondingauthor

\section{Tocitethisarticle:} \\ Saida Ed-dyb, Khadija Ait Zirri, Fatima Babokh, Raihane Bahri, Asmaa Lamrani, Nabila Soraa. Evolution of Resistance to Fluoroquinolones \\ of Uropathogenic Strains of Escherichia coli Isolated at the University Hospital of Marrakesh, Morocco. American Journal of Laboratory \\ Medicine. Vol. 5, No. 5, 2020, pp. 150-154. doi: 10.11648/j.ajlm.20200505.14
}

Received: September 19, 2020; Accepted: October 16, 2020; Published: October 27, 2020

\begin{abstract}
Background: Urinary tract infection is a common pathology in practice. The most implicated bacterial species is Escherichia coli. Fluoroquinolones occupy a privileged place among the molecules most prescribed in the treatment of these infections. Objective: determine the fluoroquinolone resistance profile of uropathogenic Escherichia coli strains at University Hospital Med VI of Marrakesh and analyze the evolution of this resistance over time in these isolates. Materials and methods: Descriptive study, over a period of 5 years (January 1, 2013 - December 31, 2017), covering all uropathogenic strains of E.coli isolated from outpatients and hospitalized at the University Hospital of Marrakesh. The samples were processed and analyzed in the Microbiology laboratory of the University Hospital. Results: During the study period, 1411 strains of uropathogenic E.coli were included. Women were mostly affected (68\%). The prevalence of urinary tract infection was $22 \%$. The number of strains studied and the resistance rates were calculated by combining the resistant strains and the intermediate strains. Resistance to ciprofloxacin increased from $16 \%$ in 2013 to $32 \%$ in 2017 . An increase with age has been demonstrated for resistance to fluoroquinolones. Extended spectrum beta-lactamase production significantly affected the resistance rate to fluoroquinolones in E.coli (24\% in C3G-sensitive strains and 56\% in ESBL strains). Conclusion: This study demonstrates the serious emergence of the resistance of uropathogenic E.coli strains to fluoroquinolones, hence the interest of revising the empirical treatment of urinary tract infections in our country and monitoring use antibiotics to control the diffusion of quinolone resistance in E.coli.
\end{abstract}

Keywords: E.coli, Fluoroquinolones, Resistance, Uropathogenic

\section{Introduction}

Urinary tract infections (UTIs) are a common cause of acute illness in common practice [1]. The microbial agents responsible are essentially Enterobacteriacae in both hospital and community settings. The most incriminated bacterial species is Escherichia coli, which accounts for 40 to $80 \%$ of isolated organisms $[1,2]$.

Fluoroquinolones (FQ) are major antibiotics in the therapeutic arsenal, used to treat many infections including urinary tract infections, because of their remarkable qualities. Indeed, these molecules have excellent intracellular and tissue diffusion, a broad antibacterial spectrum, are bactericidal on sensitive germs at the price of a perfectly satisfactory clinical tolerance; finally, their oral bioavailability is excellent [3]. In the context of the global emergence of antibiotic-resistant E.coli strains from antibiotics, the use of broader-spectrum antibiotics for even uncomplicated forms has become commonplace [1, 2]. Indeed, this resistance that has spread to fluoroquinolones has evolved considerably in recent years with alarming prevalence that can exceed $25 \%$ in strains $[3,4]$.

In Morocco, previous studies have also reported this problem of increased incidence of resistance of uropathogenic strains to antibiotics including fluoroquinolones mainly related to its excessive or inappropriate use [5,6]. Aware of the alarming problem of antimicrobial resistance, this work aims to determine the fluoroquinolone resistance profile of uropathogenic E.coli 
strains at Med VI University Hospital of Marrakech and analyze the evolution of this resistance over time. It also aims to undertake a review of the empirical treatment of urinary tract infections in our country to control the diffusion of quinolone resistance in $\mathrm{E}$. coli and to balance the impact on bacterial ecology.

\section{MaterialsandMethods}

This is a retrospective descriptive study on all uropathogenic strains of E.coli isolated in outpatients and hospitalized at the University Hospital of Marrakech over a period of five years from January 1, 2013 to December 31, 2017.

The samples were processed and analyzed in the Microbiology laboratory of the University Hospital Center. Urines were inoculated on non-selective Cystine Lactose Electrolyte Deficient (CLED) media and incubated in a normal atmosphere at $35+/-2^{\circ} \mathrm{C}$ for $+/-4 \mathrm{~h}$. Pure cultures had been identified and investigated for antibiotic sensitivity.

Bacterial identification was based on morphological, cultural and biochemical characters either by using conventional Api 20 E enterobacterial identification galleries or using the BD Phoenix ${ }^{\circledR}$ automaton.

The Antimicrobial susceptibility testing was carried out by the diffusion method of the disks in agar medium (Müller Hinton medium) and interpreted after measurement of the inhibition or liquid medium diameters by the BD Phoenix ${ }^{\circledR}$ system according to the most recent version of the Committee of the antibiogram of the French Society of Microbiology CA-SFM-EUCAST guidelines [7].

Data entry and statistical analysis were performed using the Microsoft Office Excel software.

\section{Results}

From 1st January 2013 to 31st December 2017, 28,692 urine specimens were processed at the level of the microbiology laboratory of Med VI University Hospital of Marrakech.

During the study period, 1706 strains of uropathogenic E.coli were included. The prevalence of urinary tract infection was $22 \%$. Community-acquired E.coli urinary infection was estimated at $60 \%$. Women were predominantly affected (68\%) (Figure 1).

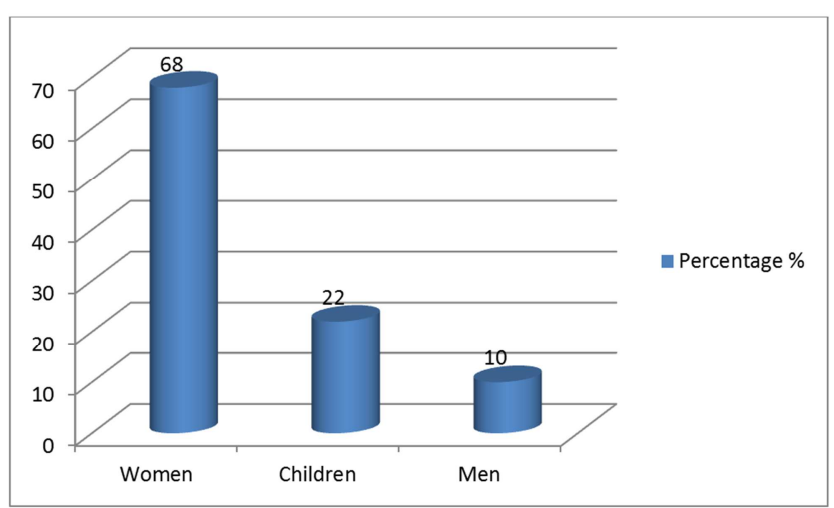

Figure 1. Prevalence of urinary tract infection in E.coli by age and sex between 2013 and 2017at the University Hospital in Marrakesh.
Male urinary tract infection accounted for only $10 \%$ of all urinary infections collected during this period. The number of strains studied and the resistance rates were calculated by combining both the resistant and intermediate strains.

Table 1 shows the evolution of resistance of uropathogenic E coli to fluroquinolones (Ciprofloxacin) from 16\% in 2013 to $32 \%$ in 2017 .

Table 1. Evolution of the resistance of uropathogenic E.coli to fluroquinolones (Ciprofloxacin) between 2013 and 2017 at the University Hospital in Marrakesh $(n=1706)$.

\begin{tabular}{ll}
\hline Year & Percentage of resistance to ciprofloxacin \\
\hline 2013 & 16 \\
2014 & 19 \\
2015 & 24 \\
2016 & 27 \\
2017 & 32 \\
\hline
\end{tabular}

An increase with age has been demonstrated for resistance to fluoroquinolones (Figure 2).

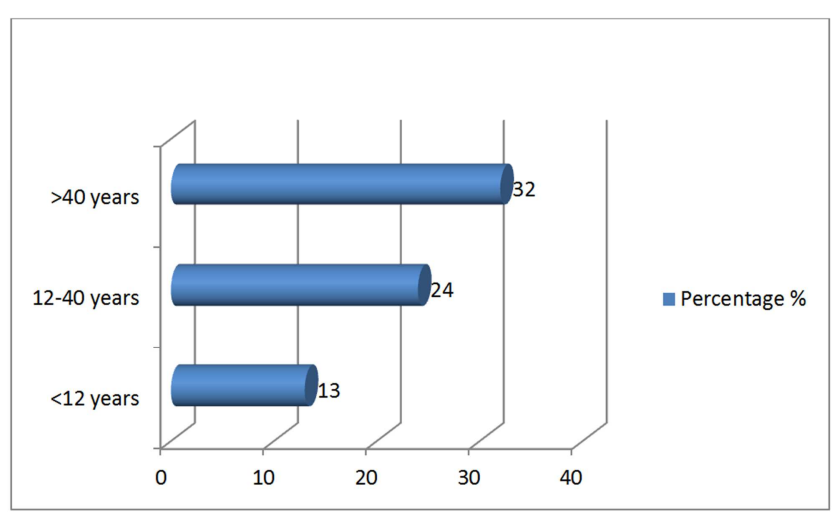

Figure 2. Age distribution of resistance of uropathogenic E.coli strains to fluroquinolones.

Table 2 reports susceptibility patterns of fluoroquinoloneresistant Escherichia coli strains between 2013 and 2017.

The antibiotics that remained the least active were amoxicillin (AMX) and cotrimoxazole (SXT) with sensitivity levels not exceeding $10 \%$ and $40 \%$, respectively..

Table 2. Antibiotic susceptibility profile of Escherichia coli strains resistant to fluoroquinolones between 2013 and 2017 at the University Hospital of Marrakesh.

\begin{tabular}{lll}
\hline Antibiotic & Number & Percentage (\%) \\
\hline Amoxicillin & 171 & 10 \\
Amoxicillin clavulanic acid & 853 & 50 \\
Cotrimoxazole & 682 & 40 \\
C3G & 682 & 40 \\
E-ESBL & 955 & 56 \\
\hline C3G: Cephalosporin third generation & \\
ESBL: Extended spectrum beta-lactamase &
\end{tabular}

Cephalosporin Third-generation resistance (C3G) was greater than $60 \%$. This decrease in $\mathrm{C} 3 \mathrm{G}$ sensitivity is mainly related to the production of extended-spectrum betalactamase which significantly impacted the resistance rate to fluoroquinolones in E.coli (24\% in C3G-sensitive strains and 
$56 \%$ in the ESBL strains.

The amoxicillin + clavulanic acid combination, however, regained efficiency with a significant increase in the level of sensitivity ranging from $21 \%$ in 2013 to $55 \%$ in 2017 .

\section{Discussion}

Urinary tract infection (UI) is the most common bacterial infection. It is a considerable economic burden on society. E. coli is by far the most frequently isolated germ. This has to do with the pathophysiology of the UI. The latter is generally ascending, and there is a strong colonization of the perineum by enterobacteria of digestive origin, and in particular E. coli [1]. In addition, there are speci fi $\mathrm{c}$ factors of uropathogenicity. E.coli has adhesins (adh. P 1 S, adh. Afa $\mathrm{M}$ ), capable of binding the bacteria to the urinary epithelium and preventing its elimination by bladder emptying [1].

The data collected during this survey provide a number of observations concerning the epidemiology of clinical E. coli strains in hospitals and communities in our context. E. coli is the first bacterial species isolated from urine samples with a percentage exceeding $60 \%$ of all isolates $[1,2]$. The noted female predominance is linked to the anatomical configuration: shortness of the urethra, proximity to the genital and anal orifices, insufficient hygiene practices, sexual intercourse and pregnancy [2].

E.coli is naturally sensitive to fluoroquinolones (FQ). Until the 1980s, the resistance of E.coli strains to FQ was low and stable (less than 20\%) [3]. the misuse of these molecules in human and veterinary medicine has increased the resistance of E. coli to FQ in recent decades [8].

This study confirms the worrying nature of the evolution of FQ resistance at E.coli in the University Hospital of Marrakech between 2013 and 2017 [5, 6]. Indeed, it highlights an evolution of clinical isolates towards resistance to FQ. This trend towards FQ resistance has increased significantly from $16 \%$ in 2013 to $32 \%$ in 2017 .

This observation is broadly consistent with the global epidemiological situation of E.coli resistance in FQ. This situation varies from country to country. In the United States, it is estimated that $10 \%$ of strains are resistant to ciprofloxacin [9]. The European situation seems to be much more worrying with resistance rates varying between 15 and $30 \%$ in Spain and France $[10,11]$. In Turkia, resistance rates of uropathogenic E. coli strains are around $40 \%[12]$.

In Morocco, Lahlou et al. found a rate of $36 \%$ [13]. In 2014, a study carried out in the urology department at Mohamed VI University Hospital in Marrakech recorded a resistance rate of $28 \%[5,6]$.

The susceptibility of community Escherichia coli strains isolated from urinary tract infections in emergency department patients is steadily decreasing and is of particular concern with regard to fluoroquinolones, calling into question the empirical treatment of acute pyelonephritis with fluoroquinolones [8].

The minimal use of quinolones in children explains high sensitivity rates to this antibiotic family which exceeded $87 \%$ in this series. Other publications have reported reassuring rates in this regard $[14,15]$.

The acquisition of resistance of uropathogenic E.coli to fluoroquinolones is a phenomenon that occurs in stages, with the gradual accumulation of "stair case" resistance mechanisms. This spread of resistance can be explained by other causes other than the clonal transmission of resistant strains. These causes are as follows:

1) The prescription of a fluoroquinolone during the last six months exposing the risk of selection of less sensitive strains: The risk of emergence of high-level resistant mutants for strains resistant to first-generation quinolones, and sensitive to fluoroquinolones [16].

2) The important ecological impact of fluoroquinolones on the gut microbiota involving a savings strategy and limits their use to specific indications [17].

3) Abuse of fluoroquinolones being the first therapeutic choice, the non-respect of the antibiotic treatment protocols by the patient and the clinician and the important shift in the practice of self-medication as a whole, with the appearance of new profiles, who practice medical diagnosis and opt for such an antibiotic which represents an irresponsible and dangerous act $[17,18]$.

4) The treatment with antibiotics without results of the Antimicrobial susceptibility testing that remains a significant approach in community [19].

The acquired resistance to these antibiotics is the result of the combination of several mechanisms: The mechanisms of resistance most frequently found are related to impermeability of the bacterial wall by reducing the expression or inactivation of the gene coding for porins. Chromosomal point mutations in antibiotic targets, gyrase (encoded by gyrA / B genes) and topoisomerase IV (parC / E gene), or the acquisition or over expression of an efflux pump reducing concentration of fluoroquinolones in bacteria [20].

However, the existence of the first levels of resistance must be investigated, either by measuring MICs or by detecting a resistance to nalidixic acid, in order to warn the clinician of the risk of decreased bactericidal activity $[3,6]$.

The association of fluoroquinolone resistance with other molecules, especially with betalactamins, is predominantly dominated by third-generation cephalosporins as we have noted has been reported by several authors [8]. This is due to frequent association between Qnr and ESBL gene determinants [21]. This Qnr gene is spreading globally with variant prevalences in Enterobacteriaceae. This highlights the possibility of co-selection of these two mechanisms of plasmid resistance. In this study, $56 \%$ of the multiresistant strains were identified (ESBL + FQ resistance). These strains of the uropathogenic E.coli studied are suspect to be carriers of both genes.

This is consistent with a study conducted in Ivory Coast which evaluated the prevalence of the "Qnr -BLSE" combination in 151 strains of ESBL-producing enterobacteria [22]. The same study reports that the percentage of ESBL 
E.coli strains with Qnr genes was 31\% [22]

\section{Conclusion}

This study demonstrates the serious emergence of the resistance of uropathogenic E.coli strains to fluoroquinolones, hence the interest of revising the empirical treatment of urinary tract infections in our country.

The prescription of fluoroquinolones must result from a very rigorous medical reasoning taking into account the national situation of antibiotic resistance due to the scale of the problem.

In view of the epidemic risk of transmitting bacterial resistance to beta-lactams and quinolones, expert authorities and societies should put in place a relevant resistance monitoring policy for better control of multiresistant strains circulation.

Given these issues, it would be imperative to undertake an action to raise public awareness through a hygiene policy and ban self-medication, to enhance scientific cooperation between biologist and clinician to rationalize the use of these drugs as well as the review of the empirical treatment of urinary tract infections in our country by promoting other therapeutic alternatives, especially in community settings, to limit inappropriate indications and by monitoring the use of antibiotics to control the spread of quinolone resistance in E.coli.

\section{References}

[1] Betsy Foxman, «Urinary Tract Infection Syndromes», Infectious Disease Clinics of North America 28, no 1 (mars 2014): 1-13

[2] Deepak S. Ipe et al., «Asymptomatic Bacteriuria: Prevalence Rates of Causal Microorganisms, Etiology of Infection in Different Patient Populations, and Recent Advances in Molecular Detection », FEMS Microbiology Letters 346, no 1 (septembre 2013): 1-10.

[3] David C. Hooper, «Mechanisms of Action of Antimicrobials: Focus on Fluoroquinolones», Clinical Infectious Diseases 32, no Supplement_1 (15 mars 2001): S9-15.

[4] V. de Lastours et B. Fantin, «Résistance aux fluoroquinolones en 2013: quel impact pour l'interniste?», La Revue de Médecine Interne 35, no 9 (septembre 2014): 601-8.

[5] S. Allali et al., «Résistance aux fluoroquinolones en urologie: prévalence, évolution et alternatives thérapeutiques à propos des principaux germes uropathogènes retrouvés entre 2004 et 2014», /data/revues/11667087/v25i13/S1166708715004029/, 29 octobre 2015 .

[6] M. C. El Bouamri et al., «Évolution récente du profil épidémiologique des entérobactéries uropathogènes productrices de $\beta$-lactamases à spectre élargi à Marrakech, Maroc», Progrès en Urologie 24, no 7 (juin 2014): 451 55 .

[7] Société Française de Microbiologie CASFM / EUCAST: Ed; 2019.
[8] B. Fantin, «Résistance aux fluoroquinolones chez les entérobactéries: quelles conséquences pour le traitement empirique ?», Antibiotiques 11, no 2 (mai 2009): 63-64.

[9] Ana C. Gales et al., «Urinary Tract Infection Trends in Latin American Hospitals: Report from the SENTRY Antimicrobial Surveillance Program (1997-2000)», Diagnostic Microbiology and Infectious Disease 44, no 3 (novembre 2002): 289-99.

[10] Eric Garnotel et al., «Sensibilité aux antibiotiques d' Escherichia coli isolé des infections urinaires communautaires: étude AFORCOPI-BIO, 2015», Revue Francophone des Laboratoires 2017, no 496 (novembre 2017): 66-73.

[11] Rosa Daza, José Gutiérrez, et Gonzalo Piédrola, «Antibiotic susceptibility of bacterial strains isolated from patients with community-acquired urinary tract infections», International Journal of Antimicrobial Agents 18, $\mathrm{n}^{\circ} 3$ (1 septembre 2001): 211-15.

[12] Hande Arslan et al., «Risk Factors for Ciprofloxacin Resistance among Escherichia Coli Strains Isolated from Community-Acquired Urinary Tract Infections in Turkey», Journal of Antimicrobial Chemotherapy 56, no 5 (1 novembre 2005): 914-18.

[13] I. Lahlou Amine, M. Chegri, et H. L' Kassmi, «Épidémiologie et résistance aux antibiotiques des entérobactéries isolées d' infections urinaires à l'hôpital militaire Moulay-Ismail de Meknès», Antibiotiques 11, no 2 (mai 2009): 90-96.

[14] A. Ferjani et al., «Caractéristiques épidémiologiques et bactériologiques des bactéries uropathogènes isolées dans un milieu pédiatrique», Archives de Pédiatrie 18, no 2 (février 2011): 230-34. A.

[15] M. Moutachakkir et al., «La résistance aux antibiotiques chez les entérobactéries uropathogènes en milieu pédiatrique au CHU de Marrakech», Journal de Pédiatrie et de Puériculture 28, no 1 (février 2015): 16-22.

[16] Eric Batard et al., «De la consommation d'antibiotiques aux résistances bactériennes: l'exemple de la résistance d'Escherichia coli aux quinolones» 17 (2011): 8.

[17] Méril Massot, Bertrand Picard, et Erick Denamur, «Diversité des populations d'Escherichia coli et leurs variations au cours $\mathrm{du}$ temps au sein du microbiote intestinal», Revue Francophone des Laboratoires 2016, no 486 (novembre 2016): 35-43.

[18] A. Hounsa, L. Kouadio, et P. De Mol, «Automédication par les antibiotiques provenant des pharmacies privées de la ville d'Abidjan en Côte d'Ivoire ", Médecine et Maladies Infectieuses 40, no 6 (juin 2010): 333-40.

[19] Gustavo A. Vasquez et al., «Risk Factors for QuinoloneResistant Escherichia Coli Urinary Tract Infection», Infectious Diseases in Clinical Practice 17, no 5 (septembre 2009): 30913.

[20] P. Nordmann et H. Mammeri, «Résistance plasmidique aux quinolones», Antibiotiques 9, no 4 (décembre 2007): 246-53.

[21] Philippe RS Lagacé-Wiens et al., «ESBL Genotypes in Fluoroquinolone-Resistant and Fluoroquinolone-Susceptible ESBL-Producing Escherichia Coli Urinary Isolates in Manitoba», Canadian Journal of Infectious Diseases and Medical Microbiology 18, no 2 (2007): 133-37. 

Isolated at the University Hospital of Marrakesh, Morocco

[22] N. Guessennd et al., «Résistance aux quinolones de type qnr chez les entérobactéries productrices de bêta-lactamases à spectre élargi à Abidjan en Côte d'Ivoire», Pathologie Biologie 56, no 7-8 (novembre 2008): 439-46. 